\title{
Giant- cell tumour of the spine with pulmonary metastases: An uncommon presentation
}

\section{Kherfani Abdelhakim ${ }^{1}$, Amri Khalil ${ }^{1}$, Khezami Karim ${ }^{1}$, Abid Leila ${ }^{2}$, Bouaziz Mouna ${ }^{3}$, Mestiri Mondher ${ }^{1}$}

\author{
${ }^{1}$ Adult surgery department, Orthopedic Institute of M T Kassab, Tunisia \\ ${ }^{2}$ Radiology department, Orthopedic Institute of M T Kassab, Tunisia \\ ${ }^{3}$ Pathologists department, Orthopedic Institute of M T Kassab, Tunisia
}

\section{Email address:}

akhalil.kh@gmail.com (A. Khalil)

\section{To cite this article:}

Kherfani Abdelhakim, Amri Khalil, Khezami Karim, Abid Leila, Bouaziz Mouna, Mestiri Mondher. Giant- Cell Tumour of the Spine with Pulmonary Metastases: An Uncommon Presentation. Journal of Surgery. Vol. 2, No. 1, 2014, pp. 14-16.

doi: $10.11648 /$ j.js.20140201.15

\begin{abstract}
Giant-cell tumour (GCT) of the bone is a relatively uncommon tumour that is classified as benign. Pulmonary metastases appear in $3 \%$ of cases, usually over three years after the first diagnosis of the tumour. The authors reports a case of 28-years-old woman, treated for a giant-cell tumour with an early pulmonary metastases.
\end{abstract}

Keywords: Spine Surgery, Giant-Cell Tumour, Pulmonary Metastases

\section{Introduction}

Giant-cell tumour (GCT) of the bone is a relatively uncommon tumour that is classified as benign.

It is known to be locally aggressive with a high recurrence rate. Pulmonary metastases appear in $3 \%$ of cases, usually over three years after the first diagnosis of the tumour, an earlier diagnosis of pulmonary metastases seems to be uncommon.

\section{Case Report}

A 28-year-old woman presented for mid-back pain with no prior symptoms. Plain radiographs, computed tomography (CT) and resonance imaging study (MRI), showed a suspicious lesion of the L1 (fig1).

A CT guided biopsy was performed, and the pathology was read as an aneurysmal bone cyst (ABC).

Two weeks later the patient presented a L1 pathological fracture secondary to the extension of the volum tumour. Facing this unusual evolution and the fast extension of the tumor, surgical treatment with a second intraoperative biopsy, was indicated. The treatment consisted on intralesional excision through a combined anterior and posterior approach with extended vertebral fixation and autologous bone graft, made after a preoperative embolization (fig 2). The new pathology tissue was examinated and interpreted as GCT with area of secondary $\mathrm{ABC}$.

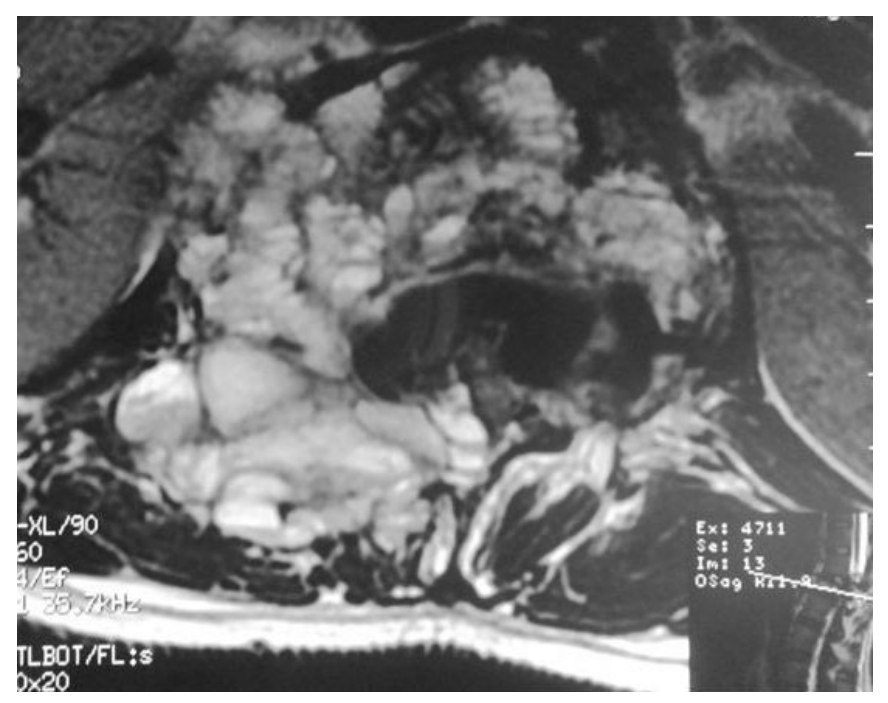

Fig 1. MRI large antéro-lateral mass of L1.

The patient recovered fully after the surgery and was able to continue her regular daily activities.

One year later, she developed progressively worsening back pain. A CT scan was performed, showing a large soft 
tissue mass postero-lateral to the spine and arising from the previously operated area with a fortuitous discovery of pulmonary multiple nodules (fig3).

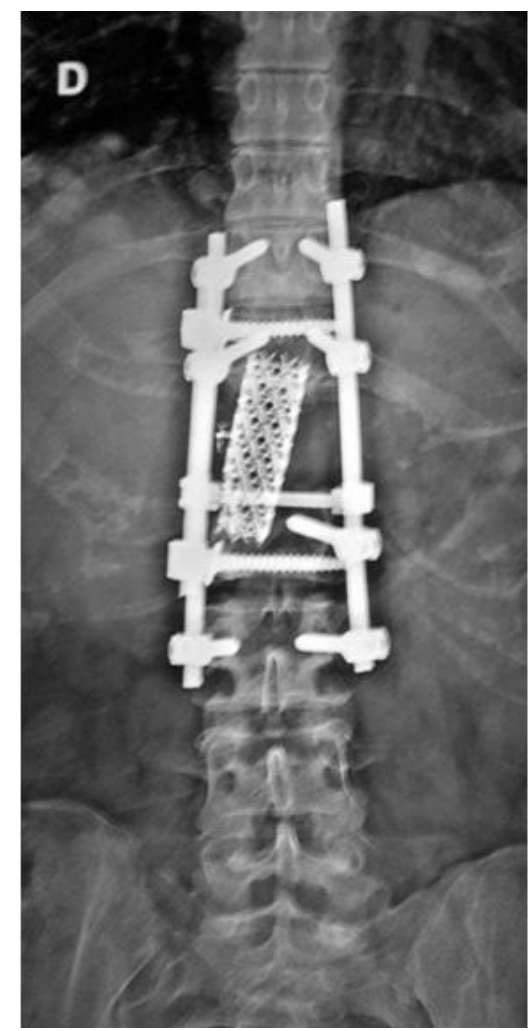

Fig2. anterior and posterior vertebral fixation.

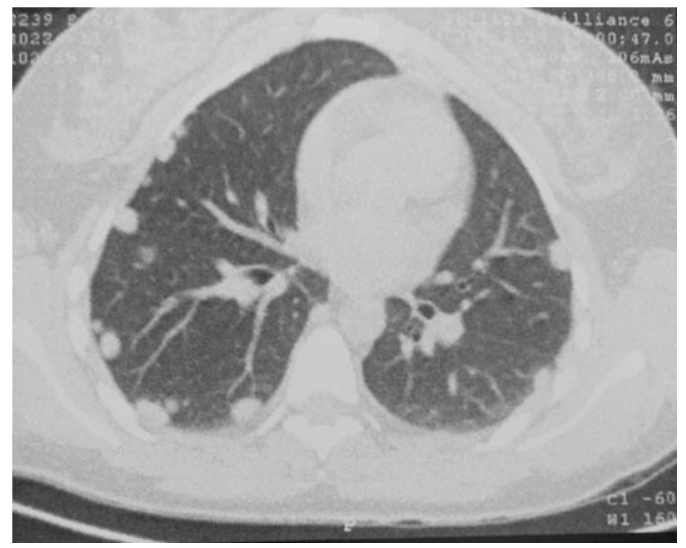

Fig3. Chest CT, multiple nodules.

The patient had a second intralesional excision through a posterior approach associated to a metastectomy of one of the lung nodules with preoperative embolization. The pathology study of the nodule confirmed the diagnosis of lung metastases of GCT. Radiation was performed for the local lesion and the patient underwent also chemotherapy.

At the latest follow-up (thirteen months), the patient is alive with the disease and is able to resume her daily activities with no respiratory symptoms. Two CT scan made at three and six months after the second surgery, showed no growth of the tumour volum.

\section{Discussion}

Giant cell tumor of the bone is a benign primary tumour with an incidence of about $5 \%$ of all the primary bone tumours [1]. The incidence in the spine ranges from 1, 4\% to 9 , and $4 \%$ [2]. The risk of metastases from a GCT is between $1,8 \%$ and $9,1 \%$ [3].

A study on the proliferative behavior based on the Ki-67 staining on a small case series showed that the most aggressive lung metastases had come from primary tumours with higher rates of proliferation [4]. Another study made by Rakesh Donthieni and al showed that the stage three of Enneking seems to have the higher risk of recurrences and metastases. A counter argument for correlation with the stage of GCT was made by Lausten et al [5] who followed up 31 consecutive patients. Their findings were that the stage of the GCT did not have any effects on the recurrences, but the type of treatment intralesional versus wide, did. Incidentally, three of their 31 patients had tumours in the vertebrae and were treated with intralesional curettage and radiation. Although treated with intralesional resection, none of these patients had a local recurrence or lung metastases, with follow-ups ranging from 198 to 278 months.

Treatment of the lung metastases includes radiation to the lungs, chemotherapy, and surgical resection, the latter, when possible, seems to be the most effective. In our case, the lung nodules were too many for a complete surgical resection. The behavior of the lung lesions is somewhat unpredictable, with a small percentage regressing with or without any adjuvant treatment.

\section{Conclusion}

GCT still one of the most controversial and discussed bone tumors, its diagnosis may be very difficult and requires a careful study of the pathology tissue.

En bloc resection when it is possible, is well known to produce the lowest recurrence rate with a higher risk of complications and neurologic deficits, intralesional excision can spare nerve roots but must be associated to radiotherapy and chemotherapy, because of the higher risk of recurrence. When there is a recurrence of GCT, with or without metastases, the local recurrence and possibly the metastases must be biopsied to confirm the original diagnosis. The behavior of the lung lesions is somewhat unpredictable, with a small percentage regressing with or without any adjuvant treatment.

\section{References}

[1] Cheng JC, Johnston JO (1997) Giant cell tumor of bone. Prognosis and treatment of pulmonary metastases. Clin Orthop Relat Res 338:205-214

[2] Sanjay BK, Sim FH, Unni KK, McLeod RA, Klassen RA. Giant-cell tumours of the spine. J Bone Joint Surg Br. 1993;75:148-154. 
[3] Bertoni F, Present D, Sudanese A, Baldini N, Bacchini P, Campanacci M (1988) Giant-cell tumor of bone with pulmonary metastases. Six case reports and a review of the literature. Clin Orthop Relat Res 237:275-285

[4] Osaka S, Toriyama M, Taira K, Sano S, Saotome K (1997)
Analysis of giant cell tumor of bone with pulmonary metastases. Clin Orthop Relat Res 335:253-61

[5] Lausten GS, Jensen PK, Schiodt T, Lund B (1996) Local recurrences in giant cell tumour of bone. Long-term follow up of 31 cases. Int Orthop 20(3):172-176 